\title{
Potassium isomerized linoleate satisfies EPA MB-35-00 for surface disinfection of Candida auris (AR-0381)
}

David Changaris, Anne Carenbauer

Microbiology, Ceela Naturals, LLC

\section{Abstract}

Four distinct lots of Potassium Isomerized Linoleate, $442 \mathrm{mM}$ or $141 \mathrm{mg} / \mathrm{ml}$ (within the cosmeticcleanser and commercial-soap acceptable concentration ranges but much greater than physiologic conditions), showed greater than 5 log kill rates (total) for Candida auris (AR-0381) for all carriers during 5 separate procedural runs of EPA MB-35-00. After exposing the inoculated stainless-steel carriers to the plant-oil soap for 1, 2, or 10 minute(s), we recovered no Candida auris on these tested carriers. Control carriers with phosphate-buffered saline applied, recovered 5.0-6.8 Log of colonyforming units per carrier.

\section{Introduction}

The United States Center for Disease Control and Prevention (CDC) identifies C. auris as a major global threat, with fatality rates in immunocompromised individuals ranging from 10-60\% [1]. Finding safe and effective non-toxic disinfectants against $C$. auris has proven difficult [2], [3], [4], [5], [6]. The plant oil Isomerized Linoleic acid has maintained Generally Recognized as Safe (GRAS) status as a food additive since 2008 [7]. Making a potassium salt of a plant oil remains a simple and readily achievable process to effect a cosmetically acceptable cleanser or soap. Current regulatory guidance from the Environmental Protection Agency (EPA) states: "In 1988, the EPA determined that soap salts have 'no independent pesticidal activity' in antimicrobial products, and must be classified as inert ingredients in those products ... Antimicrobials that still contain soap salts as active ingredients are considered misbranded...."[8]

Linoleic acid has numerous reported anti-fungal effects, including isomerized linoleic acid known to reduce hyphae formation in C. albicans ${ }^{[9]}$ (Shareck et al., 2011). Methyl esters of sunflower oils appear to inhibit Candida glabrata. And laurel seed oil and coriander seed oil show antifungal effects against the fungi type $C$. albicans ${ }^{[10](P i n t o ~ e t ~ a l ., ~ 2017) . ~ L a u r i c ~ a c i d, ~ m y r i s t o l e i c ~ a c i d, ~ l i n o l e i c ~ a c i d ~ a n d ~}$ arachidonic acid derived from bovine whey inhibits the germination of $C$. albicans «11(Cansel, 2019). Recent reports link quorum sensing Farnesol like activity to 31 saturated and unsaturated fatty acids, six medium-chain saturated fatty acids, that is, heptanoic acid, octanoic acid, nonanoic acid, decanoic acid, undecanoic acid, and lauric acid, effectively inhibited $C$. albicans biofilm formation by more than $75 \%$ at $2 \mu \mathrm{g} \mathrm{ml-1}$ with MICs in the range 100-200 $\mu \mathrm{g}$ ml-1[12](Lee et al., 2020). The many natural sources of anti-C. albicans compounds include "traditional Chinese medicine"[13](Gong et al., 2019). 
The concentration used herein, $442 \mathrm{mM}$ or $141 \mathrm{mg} / \mathrm{ml}$, falls within the range of plant oil salts generally used as commercial cosmetic cleansers or soaps [14][15] (Thompson, 2014, Ade, 2020).

\section{Objective}

To test the biocidal capacity of potassium isomerized linoleate against $C$. auris (AR-0381) using a standard biocidal test, US Environmental Protection Agency protocol MB-35-00.

\section{Results \& Discussion}

Four distinct lots of Potassium Isomerized Linoleate, $442 \mathrm{mM}$, showed greater than 5 log kill rates (total) for C. auris (AR-0381) for all carriers during 5 separate procedural runs of SOP EPA MB-3500 [16]. All aspects of the SOP were applied, including: Pegen laser-cut carriers; generation of $-80^{\circ} \mathrm{C}$ glycerol stocks; pre-application of the "soil load" containing mucin, bovine serum albumin, and yeast extract; and drying the carriers under vacuum. We processed these carriers for testing the same-day we made them. Exposing the $10 \mathrm{~mm}$ C. auris-inoculated and dried stainless steel carriers to the soap salt at 1,2 , and 10 min showed no $C$. auris recovery on the membrane filters after 120 hours of incubation on Sabourad-dextrose (Emmons) agar at $31^{\circ} \mathrm{C}$. Phosphate-buffered saline controls of the same carriers provided consistent results between 5.0-6.8 log colony forming units per carrier, when inputs of culture were controlled using OD600 between 15-19, in phosphate-buffered saline. Dilutions of 1 to 10 of the culture were used to maintain range within the UltraSpec Cell Monitor. 
bioRxiv preprint doi: https://doi.org/10.1101/2021.02.23.432518; this version posted February 23, 2021. The copyright holder for this preprint (which was not certified by peer review) is the author/funder, who has granted bioRxiv a license to display the preprint in perpetuity. It is made available under aCC-BY-ND 4.0 International license.

\section{Figures}

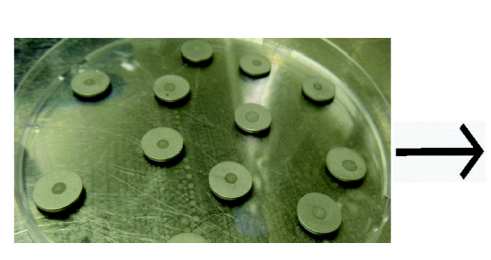

Carriers are placed in individual vial caps. 5 samples of $50 \mu \mathrm{L}$ test substance $=$ treated 3 samples of $50 \mu \mathrm{L}$ PBS applied as controls
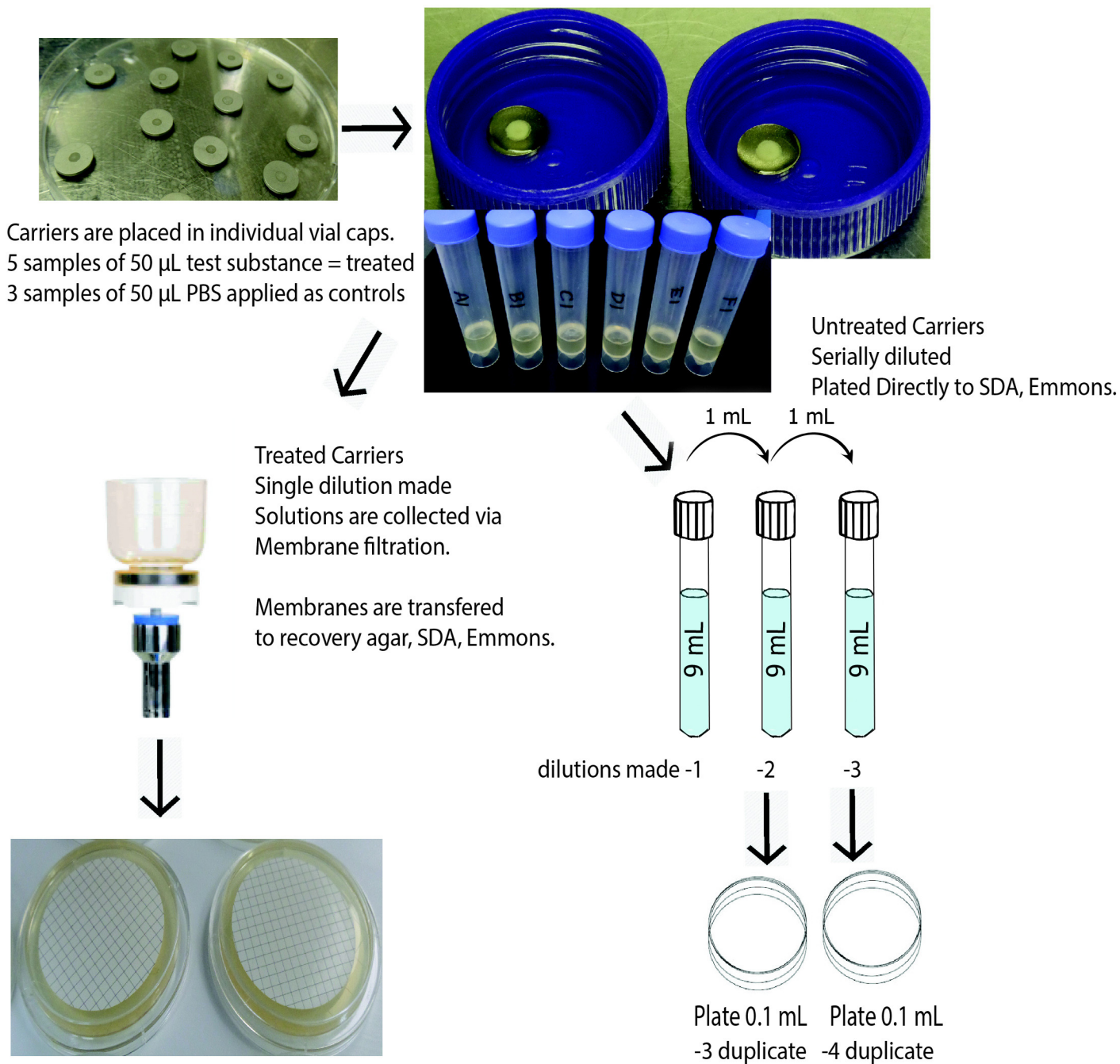

Treated Carriers Single dilution made Solutions are collected via Membrane filtration.

Membranes are transfered to recovery agar, SDA, Emmons.

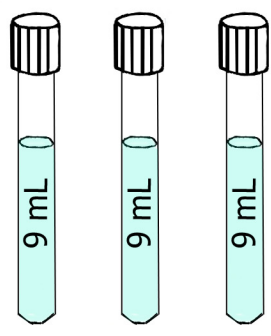

dilutions made -1

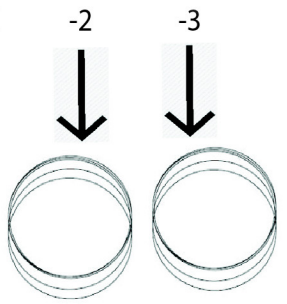

Plate $0.1 \mathrm{~mL}$ Plate $0.1 \mathrm{~mL}$ -3 duplicate -4 duplicate

Figure a. 


\section{Assay | Batch | Exposure | Control | Density | Test Disks}

$\begin{array}{rrrrrr}1 & 1 & 10 & \mathrm{n}=3 & 5.0,5.0,5.5 & \mathrm{n}=9^{*} \\ 2 & 1 & 10 & \mathrm{n}=3 & 5.7,5.7,5.5 & \mathrm{n}=7^{*} \\ 3 & 1 & 10 & \mathrm{n}=3 & 6.1,5.9,5.9 & \mathrm{n}=7^{*} \\ & 2 & 10 & & & \mathrm{n}=7^{*} \\ 4 & 1 & 2 & \mathrm{n}=3 & 5.7,6.8,5.9 & \mathrm{n}=5^{*} \\ & 2 & 2 & & & \mathrm{n}=5^{*} \\ & 3 & 2 & \mathrm{n}=3 & 5.7,5.7,5.7 & \mathrm{n}=5^{*} \\ 5 & 4 & 2 & & & \mathrm{n}=5^{*} \\ & 1 & 1 & \mathrm{n}=3 & 5.7,5.9,5.9 & \mathrm{n}=5^{*} \\ & 2 & 1 & & & \mathrm{n}=5^{*} \\ & 3 & 1 & & & \mathrm{n}=5^{*} \\ & 4 & 1 & & & \mathrm{n}=5^{*}\end{array}$

\section{*No Detectable Growth}

Figure b

\section{Figure Legend}

US EPA Protocol MB-35-00 (a) run on five separate dates testing the efficacy of potassium isomerized linoleate (442 mM or $141 \mathrm{mg} / \mathrm{ml}$ ) against Candida auris (AR-0381) documented greater than 5 log suppression with exposures at 1, 2, and 10 minutes (b). "SDA" references Saboraud dextrose agar. The EPA protocol establishes the procedure by which one satisfies the requirement of a 5-log reduction for certification. Before inoculation, we estimated by O.D. 600, that each assay parent culture of C. auris contained between 5-6 log density. The column labeled "Density" represents the "control" C. auris densities by plating (b). For example, Assay 1 reports "5.0, 5.0, 5.5." This means that within the "Control" part of Assay 1, we inoculated three separate "carries" with C. auris. We washed each "carry" with the neutralization buffer, putting each through serial dilution in PBS and plating in duplicate for counts of CFU/mL. We obtained the values of 5.0 log for two "carries" and 5.5 logs for the remaining "carry." We processed 18 "Control Carries" with cultured-plate densities of 5-6 logs, in duplicate distributed over 5 assays. For the "Experimental" or "Treated Arm" at 10-minute exposure, 30 "carries" were inoculated and subsequently treated and cultured, showing no growth of $\mathrm{C}$. 


\section{Conclusions}

In summary, we have shown that a plant oil soap salt, potassium isomerized linoleate has the germicidal capacity to effectively disinfect C. auris (AR-0381) on hard non-porous surfaces within US EPA Protocol MB-35-00.

\section{Limitations}

The mechanism of potassium isomerized linoleate biocidal activity against $C$. auris (AR-0381) remains obscure.

\section{Alternative Explanations}

The plant-oil soap may lyse the cells' walls, bind to cell membranes and ion channel proteins, or alter essential functions.

\section{Conjectures}

The central conjugated-pi bonds within the lipid may attract molecular complexes on the surface structures [17], [18]. The alpha-carboxyl salt may interact as a detergent with local hydrophilic structures while the omega aliphatic end may disorder hydrophobic surface structures. Distinct amino acid residues of the membrane ion channels may also provide Diels-Alder-adduct-affinity sites inside the channels, facilitating disruption through osmotic lysis. Signaling lipids of non-conjugated families are known to diffuse through membranes to affect nuclear mechanisms in yeast and bacteria [19], [20], [21].

We speculate that the $442 \mathrm{mM}$ or $141 \mathrm{mg} / \mathrm{ml}$ concentration is close to minimal concentration to successfully fulfill the 1 min exposure using US EPA Protocol MB-35-00.

\section{Methods}

We followed the United States Environmental Protection Agency Protocol MB-35-00 as written [16]. We dispersed Candida auris (AR-0381) obtained from the CDC AR Isolate Bank into glycerol stocks and stored at $-80^{\circ} \mathrm{C}$.

Each experiment began with an $8 \mathrm{~mL}$ overnight culture in Sabouraud dextrose broth (Emmons) from fresh $-80^{\circ} \mathrm{C}$ glycerol stock. The defined procedure included harvesting by centrifugation, resuspension in phosphate-buffered saline, and mixing with a 3-part soil load containing both with yeast extract powder (RM027, Himedia), bovine serum albumin (A2153, Sigma Life Science), and gastric mucin (HY-B2196/CS-7626, Med Chem Express). We inoculated the prepared $10 \mathrm{~mm}$ metal carriers (430-107L, Pegen Industries) and dried them in a desiccator under vacuum with a drying resin. For the treated group, we coated each disk with $50 \mu \mathrm{L} 0.442 \mathrm{M}$ Potassium Isomerized Linoleate 
onto the disk surface for 1, 2, or 10 minutes. Staggered starting times allowed for the full set of carriers. Carriers were transferred with sterile forceps to $10 \mathrm{~mL}$ neutralization solution (Sabouraud Dextrose Broth, Himedia), and vortexed for 1 minute. We filtered the entire neutralization solution plus 2 phosphate-buffered saline washes from the carriers, through membrane filters (200300-01, Rocker) with 47 mm PES 0.45 micron pore size (PES4547100, Sterlitech). We placed each membrane onto Sabouraud-dextrose-agar-(Emmons) plates and cultured for 120 hours at $31^{\circ} \mathrm{C}$.

We processed the controls similarly, using phosphate-buffered saline (50 $\mu \mathrm{L}$ ) applied to inoculated metal-disk carriers. We transferred these disks to $10 \mathrm{~mL}$ Sabouraud-dextrose-broth-neutralization solution, diluted serially in phosphate-buffered saline, and plated directly to Sabouraud-dextroseagar (Emmons) plates to culture for 120 hours at $31^{\circ} \mathrm{C}$. The MB-35-00 protocol identifies this method between treated and control samples to eliminate possible cross-contamination of the test samples in the filtration process.

\section{Funding Statement}

After Accident Care, LLC provided the funds for this study.

\section{Acknowledgements}

We thank the EPA and CDC for graciously providing the Candida auris strain AR-0381 for study.

\section{Conflict of interest}

The authors do declare conflicts of interest:

David G. Changaris is the sole owner of Ceela Naturals, LLC providing the potassium isomerized linoleate, After Accident Care, LLC providing funding for this study, and US patents concerning antimicrobial capacity of isomerized linoleic acid.

\section{Ethics Statement}

No fraudulence was committed in performing these experiments or during processing of the data. 


\section{References}

1. Us CDC Tracking Candida auris CDC 2020

2. Forsberg, Kaitlin, Woodworth, Kate, Walters, Maroya, , Vallabhaneni, Snigdha

Candida auris: The recent emergence of a multidrug-resistant fungal pathogen

Medical Mycology, 57/2019, pages 1-12 DOI: 10.1093/mmy/myy054

3. Chaabane, Farid, Graf, Artan, Jequier, Leonard, Coste, Alix T

Review on Antifungal Resistance Mechanisms in the Emerging Pathogen Candida auris

Frontiers in Microbiology, 10/2019, page 2788 DOI: 10.3389/fmicb.2019.02788

4. Fu, Leiwen, Le, Tingting, Liu, Zhihua, , Hu, Jing

Different ecacies of common disinfection methods against Candida auris and other Candida species

Journal of Infection and Public Health, 13/2020, pages 730-736 DOI: 10.1016/j.jiph.2020.01.008

5. Sexton, D. Joseph, Welsh, Rory M., Bentz, Meghan L., , Litvintseva, Anastasia P.

Evaluation of nine surface disinfectants against Candida auris using a quantitative disk carrier method:

EPA SOP MB-35 Infection Control \\& Hospital Epidemiology, 41/2020, pages 1219-1221

DOI: $10.1017 /$ ice.2020.278

6. Lockhart, Shawn R. Candida auris and multidrug resistance Dening the new normal

Fungal Genetics and Biology, 131/2019, page 103243 DOI: 10.1016/j.fgb.2019.103243

7. Center For Food Safety Applied Nutrition GRAS Notice Inventory No. GRN 0002322008

8. Us Epa EPA-738-F-92-013 1992

9. Shareck, Julie, Nantel, André, Belhumeur, Pierre

Conjugated linoleic acid inhibits hyphal growth in Candida albicans by modulating Ras1p cellular levels and downregulating TEC1 expression Eukaryotic Cell, 10/2011, page 565-577

10. Pinto, Maria E.A., Araújo, Sthéfane G., Morais, Marcela I., , Lima, Luciana A.R.S.

Antifungal and antioxidant activity of fatty acid methyl esters from vegetable oils

Anais da Academia Brasileira de Ciências, 89/2017, page 1671-1681

11. Cansel, C Antifungal Effect on Candida Albicans of Laurel, Coconut and Coriander Seed Oil

Open Access Journal of Pharmaceutical Research, 3/2019

12. Lee, Jin-hyung, Kim, Yong-guy, Khadke, Sagar Kiran, Lee, Jintae

Antibiolm and antifungal activities of medium-chain fatty acids against Candida albicans via mimicking of the quorum-sensing molecule farnesol Microbial Biotechnology, 2020, pages 17517915.13710 
13. Gong, Ying, Liu, Weiguo, Huang, Xin, , Sun, Shujuan Antifungal Activity and Potential Mechanism of N-Butylphthalide Alone and in Combination With Fluconazole Against Candida albicans

Frontiers in Microbiology, 10/2019, page 1461

14. Thompson, Jackie Liquid soapmaking: tips, techniques and recipes for creating all manner of liquid and soft soap naturally 2014

15. Ade, Sarah Self suciency soap making with natural ingredients 2016

16. Us Epa Interim Guidance for the Efficacy Evaluation of Products for Claims against Candida auris 17. Chow, Nancy A, Gade, Lalitha, Tsay, Sharon V, , Zhu, Yan Multiple introductions and subsequent transmission of multidrug-resistant Candida auris in the USA: a molecular epidemiological survey The Lancet Infectious Diseases, 18/2018, pages 1377-1384 DOI: 10.1016/S1473-3099(18)30597-8

18. Feixas, Ferran, Matito, Eduard, Poater, Jordi, , Miquel Understanding Conjugation and Hyperconjugation from Electronic Delocalization Measures The Journal of Physical Chemistry A, 115/2011, pages 13104-13113 DOI: 10.1021/jp205152n

19. Swearingen, Matthew C, Sabag-Daigle, Anice, Ahmer, Brian M Are There Acyl-Homoserine Lactones within Mammalian Intestines? Journal of Bacteriology, 195/2013, pages 173-179 DOI: 10.1128/JB.01341-12

20. Remy, Benjamin, Mion, Sonia, Plener, Laure, Elias, Mikael, , Daude, David Interference in Bacterial Quorum Sensing A Biopharmaceutical Perspective Frontiers in Pharmacology, 9/2018, page 203 DOI: 10.3389/fphar.2018.00203

21. Wongsuk, Thanwa, Pumeesat, Potjaman, Luplertlop, Natthanej

Fungal quorum sensing molecules Role in fungal morphogenesis and pathogenicity Quorum sensing in fungi Journal of Basic Microbiology, 56/2016, pages 440-447 DOI: 10.1002/jobm.201500759 